\title{
Expression of Fhit, hWAPL and Survivin in Cervical Squamous Cell Carcinoma and Significance Thereof
}

\author{
Yongmei Li ${ }^{1, ~ *, ~ Y u b i n ~ Z h a n g ~}{ }^{2}$, Lin Li ${ }^{1}$ \\ ${ }^{1}$ Department of Gynecology, Binzhou People's Hospital, Binzhou, P. R. China \\ ${ }^{2}$ Department of Nuclear Medicine, Binzhou People's Hospital, Binzhou, P. R. China
}

Email address:

liyongmei2020@163.com (Yongmei Li)

${ }^{*}$ Corresponding author

To cite this article:

Yongmei Li, Yubin Zhang, Lin Li. Expression of Fhit, hWAPL and Survivin in Cervical Squamous Cell Carcinoma and Significance Thereof. Science Journal of Public Health. Vol. 8, No. 4, 2020, pp. 108-114. doi: 10.11648/j.sjph.20200804.13

Received: May 26, 2020; Accepted: June 22, 2020; Published: June 29, 2020

\begin{abstract}
Objective To preliminarily discuss the role of fragile histidine triad (FHIT) gene, human wings apart-like (hWAPL) gene, survivin gene in lymphatic metastasis of cervical squamous cell carcinoma. Method Expression of FHIT, hWAPL and survivin in 30 cases of normal squamous epithelium, 30 cases of CIN III and 60 cases of cervical squamous cell carcinoma was detected using the immunohistochemical technique. A statistical analysis was conducted for the relationship between the expression levels of FHIT, hWAPL and survivin and clinical pathologic parameters through expression count LVD of FHIT. Results FHIT value is $1.93 \pm 0.57$ in the group of normal squamous epithelium, $2.87 \pm 0.63$ in the group of CIN III and $4.12 \pm 0.72$ in the group of cervical squamous cell carcinoma. The difference has statistical significance $(\mathrm{P}<0.05)$. The positive expression of hWAPL in normal cervical tissues is $0.00 \%(0 / 30)$, the positive expression rate is $36.67 \%(11 / 30)$ in the group of CIN III, and the positive expression rate is $63.33 \%(38 / 60)$ in the group of cervical squamous cell carcinoma. The difference has statistical significance $(\mathrm{P}<0.01)$. The positive expression rate of survivin in normal cervical tissues is $0.00 \%(0 / 30)$, the positive expression rate is $30.00 \%(9 / 30)$ in the group of CIN III, the expression rate is $61.67 \%(37 / 60)$ in the group of cervical squamous cell carcinoma. The difference has statistical significance $(\mathrm{P}<0.01)$. Conclusion FHIT, hWAPL and survivin promote lymphangiogenesis during occurrence and progression of cervical squamous cell carcinoma and lymphangiogenesis plays a vital role in lymphatic metastasis of cervical squamous cell carcinoma.
\end{abstract}

Keywords: FHIT, hWAPL, Survivin, Cervical Squamous Cell Carcinoma, Lymphatic Vessels

\section{Background}

Cervical cancer is a common malignant tumor of the female reproductive system. There are approximately 510,000 new cases and 288,000, death case all over the world. And the morbidity is rising year by year and cervical cancer commonly occurs among the young population. In China, the morbidity of cervical cancer is the highest among gynecologic malignant tumors and cervical cancer is second only to breast cancer as one of the primary causes of female death [1]. Main metastatic routes of cervical cancer include direct spreading and lymphatic metastasis. Lymphatic metastasis may occur in early-stage cervical cancer and lymphatic metastasis is the leading cause influencing treatment and prognosis of patients with cervical cancer [2]. Based on clinical statistics, The survival rate of patients with cervical cancer without lymphatic metastasis over 5 years can reach $80-90 \%$ and the survival rate of those with local or distant metastasis is only $50.9 \%$ and $16.9 \%$ [3]. Therefore, probe into the mechanism of lymphatic metastasis of cervical cancer is significant to treatment of cervical cancer and improvement of patient prognosis.

The specific mechanism of lymphatic metastasis of malignant tumor still remains unclear. It is generally accepted that tumour cells can invade into lymphatic capillaries, then aggregate to form tumor embolus and enter lymphatic vessels and regional lymph nodes at a lower level. Finally, lymphatic metastasis forms. [4]. In some researches, it is considered that lymph is identical to interstitial fluid; lymphatic vessel lumen is large and lymph has a low flow rate. Tumour cells are more likely to survive in lymphatic vessels. Basilar membranes of 
lymphatic vessels are discontinuous and No tight junction exists among endothelial cells. Therefore, tumour cells are more likely to invade into lymphatic vessel lumens through lymphatic vessel walls leading to lymphatic channel metastasis. [5]. Currently, it can be inferred that metastasis lymphatic of tumor is formed through lymphatic vessels based on the two viewpoints: First, the tumour cells invade into lymphatic vessels around tumor tissues and tumour cells enter lymphatic vessels to allow for lymphatic metastasis; second, tumour cells can induce lymphatic vessels formation thus increasing approaches for lymphatic metastasis [6]. In recent years, finding of lymphatic vessel markers, such as fragile histidine triad (FHIT) gene, human wings apart-like (hWAPL) gene, survivin gene, renal tubule foot process cell membrane mucoprotein etc., and lymphangiogenesis factor has substantially promoted related researches on lymphatic vessels [7].

In the research, expression of FHIT, hWAPL and survivin in cervical squamous cell carcinoma tissues was detected using the immunohistochemical technique to preliminarily discuss the role of FHIT, hWAPL and survivin in lymphatic metastasis of cervical squamous cell carcinoma.

\section{Material and Methods}

\subsection{Object of Study}

Extensive uterus resection + pelvic lymph node dissection were performed for 60 cases with cervical squamous cell carcinoma at our hospital from May, 2012 to May, 2013. Cervical paraffin specimens demonstrated as cervical squamous cell carcinoma based on postoperative pathology was classified as the cervical squamous cell carcinoma group. All patients had complete and clinical data and did not received any radiotherapy and chemotherapy. 30 cervical paraffin specimens with uterus resected due to CIN III during the corresponding period is classified as the CIN III group. Another 30 normal Cervical paraffin specimens with uterus resected due to hysteromyoma surgery during the corresponding period is classified as the control group for research.

\subsection{Material and Instrumentation}

Rabbit Anti Human FHIT, Rabbit Anti Human hWAPL and Rabbit Anti Human survivin were purchased from U.S. Fermentas Company; immumohistochemical staining kit were purchased from Hangzhou Biowish biotechnology Co. Ltd.; DAB, PBS and citrate buffer were purchased from Shanghai Boyan Biotechnology Co., Ltd. The microscope was purchased from U.S. Corvallis Scientific Instrument and Equipment Co. Ltd.

\subsection{Tissue Processing}

The paraffin block was sectioned in $5 \mu \mathrm{m}$ thickness continuously with a paraffin slicing machine and the sections were placed in the oven at $60^{\circ} \mathrm{C}$ overnight. The sections were taken out and placed into the dimethylbenzene solution I for dewaxing for 10 minutes and then placed into the dimethylbenzene solution II for dewaxing for 10 minutes. The tissue sections hydrated and dewaxed were placed on a high-temperature-resistant staining rack and placed into boiling sodium citrate buffer for high-pressure antigen retrieval. The glass slide was taken out after 30min and washed with distilled water for 5 minutes $\mathrm{X} 2$ times and with PBS for 5 minutes X 2 times. It was incubated in $3 \% \mathrm{H}_{2} \mathrm{O}_{2}$ at room temperature for 30 minutes to eliminate activity of endogenous peroxidase and then washed with PBS for 2 minutes X3 times. It was stored in the heat preservation box at $4^{\circ} \mathrm{C}$ and incubated in the refrigerator overnight after primary antibodies were added dropwise. It was washed with PBS for 3 minutes X 3 times. Fresh prepared DAB color development solution was instilled. The staining time should be controlled based on color development under the microscope. The reaction should be terminated subject to sufficient positive color development without interference of variegated colors in the background. It was counterstained with hematoxylin for 30 minutes and washed sufficiently. It was destained with $1 \%$ hydrochloric acid differentiating solution for several seconds, washed sufficiently with water and dehydrated with ascending gradient alcohol. It was cleared in the dimethylbenzene solution for 5 minutes and observed under the microscope after being sealed with neutral gum. In each group, positive sections provided by the reagent company served as the positive control and PBS served as the blank control instead of primary antibodies.

\subsection{Determination of Immunohistochemical Staining Results}

Comprehensive scoring was performed based on staining intensity and percentage of positive cells. Scoring was performed based on gradation of specific staining present in tumour cells: colourless, 0 point, faint yellow, 1 point, brownish orange, 2 points, sepia, 3 points; percentage of positive cells was scored: negative, 0 point, positive cells $\leq 10 \%, 1$ point, positive cells $11 \%-50 \%, 2$ points, positive cells $51 \%-75 \%, 3$ points, positive cells $>75 \%, 4$ points. The immunoreaction will be deemed negative if the product of staining intensity and score of percentage of positive cells $>3$. Two persons will be arranged for respective judgement and a third party will be requested in case of any dispute [8].

\subsection{Calculating Lymphatic Vessel Density (LVD)}

FHIT positive staining was expressed in a tubular structure. Lymphatic vessel density (LVD) was calculated with FHIT positive expression. The lymphatic vessels were counted with the Weidner method: the sections were observed comprehensively under a low power microscope to determine the site with the highest destiny in the lymphatic vessels, i.e. hotspot. Then the number of lymphatic vessels with hotspots being stained under a high power microscope. As for the counting criteria, a single stained positive endothelial cell or endothelial cell cluster was counted as a positive lymphatic vessel with 5 fields for each count. The average value was 
regarded as the density of the lymphatic vessels.

\subsection{Statistical Analysis}

The SPSS 15.0 statistical method was used for analysis. The measurement data was analysed with the t test or one-way variance. Enumeration data was tested. Correlation analysis was conducted with Sperman correlation analysis. If $\mathrm{P}<0.05$ the difference has statistical significance.

\section{Results}

\subsection{Clinical Pathological Data}

The group of cervical squamous cell carcinoma: aged 33-62, average age $49.16 \pm 12.92$. Among the group, 28 cases in Stage I, 32 cases in Stage II; 35 case with lymphatic metastasis, 25 cases with lymphatic metastasis; 23 cases with tumor diameter $>4 \mathrm{~cm}$ and 37 cases with tumor diameter $\leq 4 \mathrm{~cm}$. The group of CIN III: aged 30-61, average age $47.26 \pm 11.64$. The group of normal cervix: aged 31-60, average age $48.61 \pm 11.95$.
The age difference among the three groups has no statistical significance.

\subsection{Expression of FHIT in Cervical Tissues and LVD Value}

FHIT is mainly expressed in lymphatic endothelial cells and positive stained lymphatic vessels exhibit a brownish yellow color. Morphology of lymphatic vessels in squamous epithelial tissues of a normal cervix is regular. Lymphatic vessels in cervical squamous cell carcinoma tissues are mainly distributed around tumor tissues with irregular morphology and expanded lumens. Lumens of lymphatic vessels within the tumor tissues are small with strip or cluster-shaped structure. LVDs of different cervical tissues were calculated based on FHIT positive expression. LVD value is $1.93 \pm 0.57$ in the group of normal squamous epithelium, $2.87 \pm 0.63$ in the group of CIN III and $4.12 \pm 0.72$ in the group of cervical squamous cell carcinoma. The difference has statistical significance $(\mathrm{P}<0.05)$. See Table 1 and Figure 1.

Table 1. LVDs of Different Cervical Tissues.

\begin{tabular}{lll}
\hline Group & Number of Cases & LVD Value \\
\hline Normal Cervical Tissues & 30 & $1.93 \pm 0.57$ \\
CIN III Group & 30 & $2.87 \pm 0.63 *$ \\
Group of Cervical Squamous Cell Carcinoma & 60 & $4.12 \pm 0.72 * \#$ \\
\hline
\end{tabular}

Legend: Compared with the normal cervix, ${ }^{*} \mathrm{P}<0.05$; compared with CIN III, \#P<0.05.
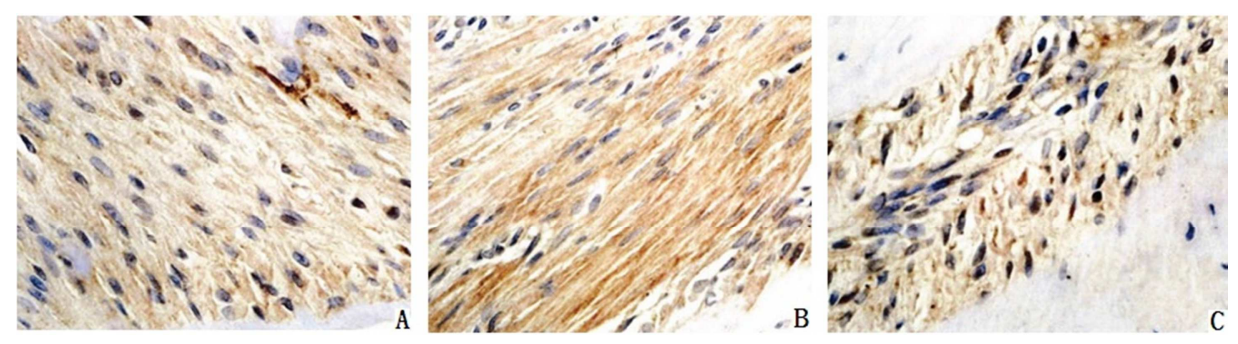

Figure 1. Expression of FHIT in Cervical Tissues.

Legend: A. Expression of FHIT in normal cervical tissues; B. Expression of FHIT in CIN III; C. Expression of FHIT in cervical squamous cell carcinoma tissues.

\subsection{Analysis on Clinical Pathological Parameter Relationship Between LVD and Cervical Squamous Cell Carcinoma}

LVD in the group of Stage I cervical squamous cell carcinoma is $5.11 \pm 1.37$ and $6.25 \pm 1.43$ in the group of Stage II cervical squamous cell carcinoma. The difference has statistical significance $(\mathrm{P}<0.05)$. LVD is $7.38 \pm 1.82$ in the group with lymph node metastasis and $5.72 \pm 1.66$ in the group without lymph node metastasis. The difference has statistical significance $(\mathrm{P}<0.05)$. LVD is $6.92 \pm 1.83$ in the poorly differentiated group, $4.36 \pm 1.71$ in the moderately differentiated group and $2.62 \pm 1.48$ in the highly differentiated group. The difference has statistical significance $(\mathrm{P}<0.05)$. LVD is $6.08 \pm 2.07$ in the group with diameter of tumor $>4 \mathrm{~cm}$ and $7.72 \pm 2.11$ in the group with diameter of tumor $\leq 4 \mathrm{~cm}$. The difference has statistical significance $(\mathrm{P}<0.05)$. See Table 2 .
Table 2. Clinicopathological Parameter of LVD and Cervical Squamous Cell Carcinoma.

\begin{tabular}{lll}
\hline Clinicopathological Parameter & Number of Cases & LVD Value \\
\hline Clinical Stages & & \\
Stage I & 28 & $5.11 \pm 1.37$ \\
Stage II & 32 & $6.25 \pm 1.43$ \\
Lymph Node Metastasis & & \\
Yes & 35 & $7.38 \pm 1.82$ \\
No & 25 & $5.72 \pm 1.66$ \\
Pathological Grading & & \\
Poorly Differentiated & 36 & $6.92 \pm 1.83$ \\
Moderately Differentiated & 17 & $4.36 \pm 1.71 *$ \\
Highly Differentiated & 7 & $2.62 \pm 1.48 * \#$ \\
Tumor Size & & \\
$>4 \mathrm{~cm}$ & 23 & $6.08 \pm 2.07$ \\
$\leq 4 \mathrm{~cm}$ & 37 & $7.72 \pm 2.11$ \\
\hline
\end{tabular}

Legend: Compared with the poorly differentiated group, $* \mathrm{P}<0.05$; compared with the moderately differentiated group, $\# \mathrm{P}<0.05$. 


\subsection{Expression of hWAPL in Cervical Tissues}

hWAPL is primarily expressed in cervical squamous cell carcinoma or cervical intraepithelial neoplasias cell cytoplasm and positive staining exhibits light yellow to reddish brown granular shape. hWAPL hardly has positive staining or exhibits weak staining in normal cervical squamous epithelial tissues. Based on immunohistochemical criteria, the positive expression is $0.00 \%(0 / 30)$, the rate of positive expression is $36.67 \%(11 / 30)$ in the group of CIN III and $63.33 \%(38 / 60)$ in the group of cervical squamous cell carcinoma. The difference has statistical significance $(\mathrm{P}<0.01)$. See Table 3 and Figure 2.

Table 3. Expression of $h W A P L$ in Different Cervical Tissues.

\begin{tabular}{llll}
\hline Group & Number of Cases & Positive Expression & Positive Rate (\%) \\
\hline Normal Cervical Tissues & 30 & 0 & 0.00 \\
CIN III Group & 30 & 11 & 36.67 \\
Group of Cervical Squamous Cell Carcinoma & 60 & 38 & 63.33 \\
P Value & 0.000 & & \\
\hline
\end{tabular}

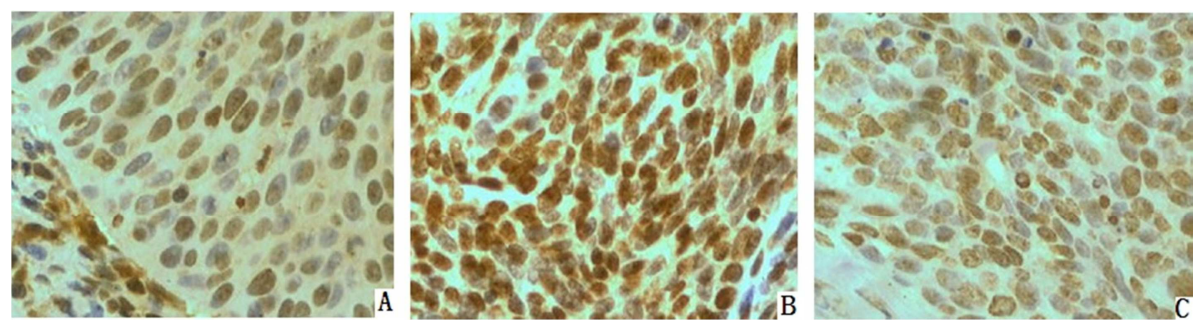

Figure 2. Expression of hWAPL in Cervical Tissues.

Legend: A. Expression of hWAPL in normal cervical tissues; B. Expression of hWAPL in CIN III; C. Expression of hWAPL in cervical squamous cell carcinoma tissues.

\subsection{Analysis on Clinical Pathological Parameter Relationship Between hWAPL Expression and Cervical Squamous Cell Carcinoma}

Based on the disease extent of tumor tissues, cervical cancer was divided into four stage clinically. In the research, cervical paraffin specimens of Stage I and II cervical squamous cell carcinoma treated directly with a surgery without any other therapeutic intervention were selected for research. Expression of hWAPL was different in different clinical stages. The positive expression rate of hWAP in the group of Stage I cervical squamous cell carcinoma is $46.43 \%$ and $75.00 \%$ in the group of Stage II cervical squamous cell carcinoma. The difference has statistical significance $(\mathrm{P}<0.05)$. The positive expression rate of hWAP is $74.29 \%$ in the group with lymph node metastasis and $48.00 \%$ the group without lymph node metastasis. The difference has statistical significance $(\mathrm{P}<0.05)$. The positive expression rate of $\mathrm{hWAP}$ is $77.78 \%$ in the poorly differentiated group and $41.67 \%$ in the moderately and highly differentiated group. The difference has statistical significance $(\mathrm{P}<0.05)$. The positive expression rate of hWAP is $82.61 \%$ in the group with tumor diameter $>4 \mathrm{~cm}$ and $56.56 \%$ in the group with tumor diameter $\leq 4 \mathrm{~cm}$. The difference has statistical significance $(\mathrm{P}<0.05)$. See Table 4.

Table 4. Expression of hWAP and Clinicopathological Parameters of Cervical Squamous Cell Carcinoma.

\begin{tabular}{|c|c|c|c|c|}
\hline Clinicopathological Parameter & Number of Cases & Negative Expression & Positive Rate (\%) & P Value \\
\hline \multicolumn{5}{|l|}{ Clinical Stages } \\
\hline Stage I & 28 & 13 & 46.43 & \multirow{3}{*}{0.013} \\
\hline Stage II & 32 & 24 & 75.00 & \\
\hline \multicolumn{4}{|l|}{ Lymph Node Metastasis } & \\
\hline Yes & 35 & 26 & 74.29 & \multirow{2}{*}{0.019} \\
\hline No & 25 & 12 & 48.00 & \\
\hline \multicolumn{5}{|l|}{ Pathological Grading } \\
\hline Poorly Differentiated & 36 & 28 & 77.78 & \multirow{3}{*}{0.008} \\
\hline Moderately and Highly differentiated & 24 & 10 & 41.67 & \\
\hline \multicolumn{4}{|l|}{ Tumor Size } & \\
\hline$>4 \mathrm{~cm}$ & 23 & 19 & 82.61 & \multirow{2}{*}{0.021} \\
\hline$\leq 4 \mathrm{~cm}$ & 37 & 20 & 55.56 & \\
\hline
\end{tabular}

\subsection{Expression of Survivin in Cervical Tissues}

Survivin is primarily expressed in cervical squamous cell carcinoma or cervical intraepithelial neoplasias cell karyon and seldom expressed in cytoplasm, and positive staining exhibits light yellow to reddish brown granular shape. Survivin hardly has positive staining or exhibits weak staining in normal cervial squamous epithelial tissues. Based on immunohistochemical criteria, the positive expression is $0.00 \%(0 / 30)$, the rate of positive expression is $30.00 \%(9 / 30)$ 
in the group of CIN III and $61.67(37 / 60)$ in the group of cervical squamous cell carcinoma. The difference has

statistical significance $(\mathrm{P}<0.01)$. See Table 5 and Figure 3 .

Table 5. Expression of survivin in Different Cervical Tissues.

\begin{tabular}{llll}
\hline Group & Number of Cases & Negative Expression & Positive Rate (\%) \\
\hline Normal Cervical Tissues & 30 & 0 & 0.00 \\
CIN III Group & 30 & 9 & 30.00 \\
Group of Cervical Squamous Cell Carcinoma & 60 & 37 & 61.67 \\
P Value & 0.000 & & \\
\hline
\end{tabular}

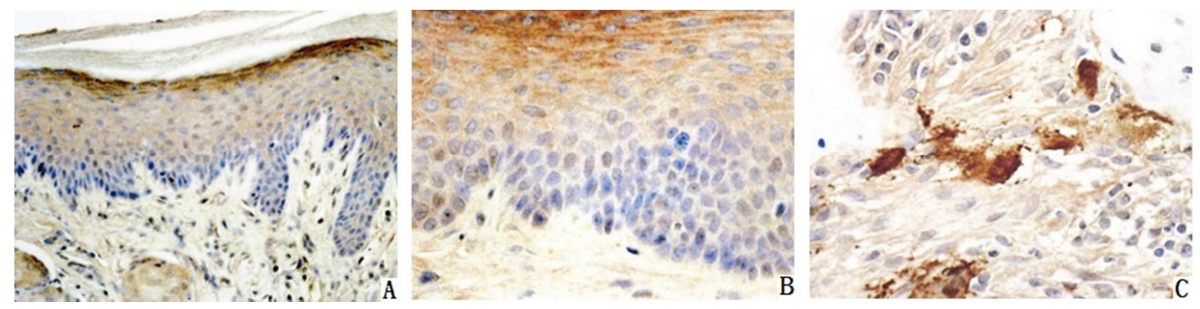

Figure 3. Expression of survivin in Cervical Tissues.

Legend: A. Expression of survivin in normal cervical tissues; B. Expression of survivin in CIN III; C. Expression of survivin in cervical squamous cell carcinoma tissues.

\subsection{Analysis on Relationship Between Survivin Expression and Clinical Pathological Parameters of Cervical Squamous Cell Carcinoma}

The positive expression rate of survivin is $53.57 \%$ in the group of Stage I cervical squamous cell carcinoma and $81.25 \%$ in the group of Stage II cervical squamous cell carcinoma. The difference has statistical significance $(\mathrm{P}<0.05)$. The positive expression rate is $82.86 \%$ in the group with lymph node metastasis and $52.00 \%$ in the group without lymph node metastasis. The difference has statistical significance $(\mathrm{P}<0.05)$. The positive expression rate is $55.56 \%$ in the poorly differentiated group and $45.83 \%$ in the moderately and highly differentiated group. The difference has no statistical significance $(\mathrm{P}>0.05)$. The positive expression rate is $78.26 \%$ in the group with tumor diameter $>4 \mathrm{~cm}$ and $48.65 \%$ in the group with tumor diameter $\leq 4 \mathrm{~cm}$. The difference has statistical significance $(\mathrm{P}<0.05)$. See Table 6 .

Table 6. Expression of Survivin and Clinicopathological Parameters of Cervical Squamous Cell Carcinoma.

\begin{tabular}{llcc}
\hline Clinicopathological Parameter & Number of Cases & Negative Expression & Positive Rate (\%) \\
\hline Clinical Stages & & & P Value \\
Stage I & 28 & 15 & 53.57 \\
Stage II & 32 & 26 & 81.25 \\
Lymph Node Metastasis & & & 0.022 \\
Yes & 35 & 29 & 82.86 \\
No & 25 & 13 & 52.00 \\
Pathological Grading & & & 0.019 \\
Poorly Differentiated & 36 & 20 & 55.56 \\
Moderately and Highly differentiated & 24 & 11 & 45.83 \\
Tumor Size & & & \\
$>4 \mathrm{~cm}$ & 23 & 18 & 0.267 \\
$\leq 4 \mathrm{~cm}$ & 37 & 18 & 78.26 \\
\hline
\end{tabular}

\section{Discussion}

Fragile histidine triad (FHIT) gene a gene located in chromosome 3 p142 region, with full length of approximately $500 \mathrm{~kb}$. FHIT coded protein is a typical diadenosine triphosphoric acid (Ap3A) hydrolase. Hydrolytic Ap3A makes relevant protein kinase activity become lower thus influencing the pathway of growth signals and inhibiting cell growth [9-10]. Some research results have show that the abnormal expression rate of FHIT exhibits an increasing trend during the process of normal cervix-cervical intraepithelial neoplasias-infiltrating carcinoma. Cervical cancer is susceptible to effect of external cancerogen and causes the gene's fragile site to rupture and decreasing or loss of FHIT protein expression. Precancerous lesions form and develop into infiltrating carcinoma after the cells obtain growth advantages. It indicates that FHIT gene plays a vital role in occurrence of cervical cancer as a cancer suppressor gene [11-12].

All of the existing researches support that new lymphatic vessels exist within and surround the tumor tissues [13]. It is still controversial whether metastasis of tumour cells occurs through lymphatic vessels in the vicinity of tumor or through lymphatic vessels within the tumor; some scholars hold that the surrounding tissues of the tumor have many lymphatic 
vessels largely in a expansive state contributing to lymphatic metastasis of tumour cells; however, metastasis will not occur to lymphatic vessels due to the fact that they largely collapse and lose their functions as a result of hydrostatic pressure within the tumor [14]. Xiong and others analysed LVD value in the cervical squamous cell carcinoma tissue utilizing immunohistochemistry. The result has shown that LVD adjacent to the tumor relates to neoplasm staging, lymphatic vessel invasion and lymph node metastasis [15]. Lee and others have found within head and neck neoplasm there are lymphatic vessels with a small lumen. Some exhibit a lacuna shape and the morphology is different from lymphatic vessels in the vicinity of the tumor tissues. The number of lymphatic vessels within the tumor relates to tumor lymphatic metastasis It was considered that malignant tumor is able to induce lymphangion genesis within the tumor and is related to tumor lymphatic metastasis [16]. Balsat and others have detected LVD marked by FHIT in tissue in 41 cases of cervical cancer and 12 cases of normal cervix and found that lymphatic vessels are observed within and around the tumor tissue and LVD is significantly higher than that of a normal cervix, which relates to lymph node metastasis and lymphatic vessel infiltration of cervical cancer, indicating that lymphatic vessels both within and around the tumor tissue play a vital role in lymph node metastasis of tumour cells [17]. In the research, SP immumohistochemical staining was performed for FHIT in normal cervical squamous epithelial tissue, CIN III and cervical squamous cell carcinoma paraffin tissue sections and it was found that the morphology of lymphatic vessels in normal cervical squamous epithelial tissue was regular while the morphology of lymphatic vessels in CIN III and tissue of cervical squamous cell carcinoma was irregular with an expansive lumen, small lumens of lymphatic vessels within tumor tissues exhibiting a strip or cluster-shaped structure. The lymphatic vessels are abundant in the marginal zone of cervical squamous cell carcinoma tumor and become smaller and have narrow lumens within interior tumor.

Human wings apart-like (hWAPL) gene is gene that have be found to closely relate to cervical cancer and HPV in recent years. It is a homologous sequence of wings apart-like (WAPL) gene in drosophilae, located at 10q231, with a length of approximately $30793 \mathrm{bp}$. It encodes a polymeric anchorin and dissociates polymerization of the chromosome arm duly in the earlier stage of mitosis. However, its overexpression can enable the sister chromatid to dissociate too early [18-19]. Some scholars have detected the expression levels of mRNA of the hWAPL in cervical cancer, ovarian cancer, breast cancer, lung cancer, renal carcinoma, colon cancer and their corresponding normal tissues and found that the mRNA expression of the hWAPL gene in cervical cancer increased significantly compared with that in other cancerous tissues and their corresponding normal tissues [20-21]. Researches also have found that expression of hWAPL in cervical cancer cells with positive and negative HPV was high but expression of hWAPL in the normal cervical tissue was lower [22]. Hence, hWAPL expression may be more closely related to cervical cancer compared with HPV infection [23]. The current researches have shown that the hWAPL gene is a high-expression gene for cervical cancer specificity [24]. In the research, the relationship between expression of hWAPL in normal cervical squamous epithelium, CIN III and cervical squamous cell carcinoma tissues, expression of HWAPL and the clinicopathologic characteristics of cervical squamous cell carcinoma (clinical stages, lymphatic metastasis, pathological grading and tumor size).

\section{Conclusion}

The survivin gene is located on chromosome 17q25 and its encoded survivin protein is a apoptosis inhibitory factor with significant effect discovered currently. Baculovirus anti-apoptosis protein repetitive sequence (bacu-loviral IAP repeat, BIR) and caspase 3, 7 and 9 are combined to inhibit apoptosis. It is located on the centromere and central spindle to regulate cell cycle [25-26]. Survivin exhibits high expression in most tumor tissues and relates to course development, prognosis and recurrence of many tumors [27]. $\mathrm{Lu}$ and others has detected expression of survivin in 30 cases of invasive cervical cancer, 15 cases of precancerous lesions and 10 cervicitis utilizing the immunohistochemistry technology and the real-time fluorescence quantification PCR technique. The result has indicated that the survivin protein and survivin mRNA shows only little expression in cervicitis tissue and shows significantly increased expression in sequence in precancerous lesions of uterine cervix and cervical cancer tissue $(\mathrm{P}<0.05)$. The quantity of expressed survivin mRNA in precancerous lesions of uterine cervix and invasive cervical cancer tissue is 11.0 times and 22.6 times of the cervicitis group respectively [28]. Some researches have further shown that expression of survivin is not relevant to age of patients and types of tumor growth but it has significant difference between different clinical stages and pathological grades $(\mathrm{P}<0.05)$. The later the clinical stage of cervical cancer is, the higher the pathological grade will be and the stronger the expression of survivin will be [29]. It indicates that survivin is closely related to genesis and progression and is a specific tumor marker with clinical significance.

\section{References}

[1] Yang W. Kim Y. et al. Rotating-shield brachytherapy for cervical cancer [J]. Phys Med Biol, 2019, 58 (11): 3931-41.

[2] Sogukpinar N. Saydam BK. et al. Assessment of Cervical Cancer Risk in Women between 15 and 49 Years of Age: Case of Izmir [J]. Asian Pac J Cancer Prev, 2019, 14 (3): 2119-25.

[3] Pesee M. Kirdpon W. et al. Palliative treatment of advanced cervical cancer with radiotherapy and thai herbal medicine as supportive remedy - analysis of survival [J]. Asian Pac J Cancer Prev, 2013, 14 (3): 1593-6.

[4] Speiser D. Kohler C. et al. Radical vaginal trachelectomy: a fertility-preserving procedure in early cervical cancer in young women [J]. Dtsch Arztebl Int, 2019, 110 (17): 289-95. 
[5] Maver PJ. Seme K. et al. Cervical cancer screening practices in central and eastern Europe in 2012 [J]. Acta Dermatovenerol Alp Panonica Adriat, 2013, 22 (1): 7-19.

[6] Bhaumik S. India has world's highest number of cervical cancer deaths [J]. BMJ, 2013, 346 (17): 3108-10.

[7] Sicam RV. Huang KG. et al. Maintenance of ovarian function in end-of-life cervical cancer patient following primary surgico-radiotherapy and ovarian transposition $[\mathrm{J}]$. J Gynecol Oncol, 2013, 24 (2): 204-7.

[8] Ushijima K. Fujiyoshi K. et al. Concurrent chemoradiotherapy with low-dose daily cisplatin for high risk uterine cervical cancer: a long-term follow-up study [J]. J Gynecol Oncol, 2019, 24 (2): 108-13.

[9] Bai H. Ge S. et al. Hypoxia inducible factor-1alpha-mediated activation of survivin in cervical cancer cells [J]. J Obstet Gynaecol Res, 2019, 39 (2): 555-63.

[10] Kasuya G. Ogawa K. et al. Postoperative radiotherapy for uterine cervical cancer: impact of lymph node and histological type on survival [J]. Anticancer Res, 2013, 33 (5): 2199-204.

[11] Shen SN. Wang LF. et al. Upregulation of microRNA-224 is associated with aggressive progression and poor prognosis in human cervical cancer [J]. Diagn Pathol, 2019, 8 (1): 69-72.

[12] Poliquin V. Decker K. et al. Changes in cervical cancer screening behavior for women attending Pap Test Week clinics [J]. Int J Womens Health, 2019, 5 (1): 141-8.

[13] Kai-Jiang L. Xin-Wei L. et al. Application of carbon nanoparticles in the laparoscopic sentinel lymph node detection in patients with cervical cancer [J]. Zhongguo Yi Xue Ke Xue Yuan Xue Bao, 2019, 35 (2): 150-4.

[14] Biedka M. Makarewicz R. et al. Labeling of microvessel density, lymphatic vessel density and potential role of proangiogenic and lymphangiogenic factors as a predictive/prognostic factors after radiotherapy in patients with cervical cancer [J]. Eur J Gynaecol Oncol, 2019, 33 (4): 399-405.

[15] Xiong Y. Cao LP. et al. Clinical significance of peritumoral lymphatic vessel density and lymphatic vessel invasion detected by D2-40 immunostaining in FIGO Ib1-IIa squamous cell cervical cancer [J]. Cell Tissue Res, 2019, 348 (3): 515-22.

[16] Lee TS. Kang SB. et al. Chemoradiation with Paclitaxel and Carboplatin in high-risk cervical cancer patients after radical hysterectomy: a korean gynecologic oncology group study [J]. Int J Radiat Oncol Biol Phys, 2019, 86 (2): 304-10.
[17] Balsat C. Blacher S. et al. Whole slide quantification of stromal lymphatic vessel distribution and peritumoral lymphatic vessel density in early invasive cervical cancer: a method description [J]. ISRN Obstet Gynecol, 2019, 35 (5): 861-3.

[18] Cao CX. Ma J. et al. Immunization of mice with plasmids coexpressing HPV16 E5 and the novel oncogene hWAPL [J]. Int J Gynecol Cancer, 2019, 18 (3): 534-9.

[19] Cao LX. Shen ZF. et al. Research progress about Wapl gene [J]. Sheng Li Ke Xue Jin Zhan, 2010, 4 (1): 27-30.

[20] Puranik AD. Purandare NC. et al. Isolated mandibular condylar metastases: An uncommon manifestation of recurrent cervical cancer [J]. J Cancer Res Ther, 2019, 9 (1): 108-10.

[21] Gao Y. Gao F. et al. Treatment results of incomplete chemoradiotherapy in locally advanced cervical cancer [J]. Onco Targets Ther, 2013, 6 (1): 297-302.

[22] Oikawa K. Akiyoshi A. et al. Expression of various types of alternatively spliced WAPL transcripts in human cervical epithelia [J]. Gene, 2018, 423 (1): 57-62.

[23] Alsbeih G. Al-Harbi N. et al. HPV prevalence and genetic predisposition to cervical cancer in Saudi Arabia [J]. Infect Agent Cancer, 2019, 8 (1): 15-7.

[24] Laudadio J. Human papillomavirus detection: testing methodologies and their clinical utility in cervical cancer screening [J]. Adv Anat Pathol, 2018, 20 (3): 158-67.

[25] Jeong BK. Huh SJ. et al. Prognostic value of different patterns of squamous cell carcinoma antigen level for the recurrent cervical cancer [J]. Cancer Res Treat, 2018, 45 (1): 48-54.

[26] Chen ZY. Liang K. et al. Study of the UTMD-Based Delivery System to Induce Cervical Cancer Cell Apoptosis and Inhibit Proliferation with shRNA targeting Survivin [J]. Int J Mol Sci, 2019, 14 (1): 1763-77.

[27] Huang Z. Mayr NA. et al. Characterizing at-Risk Voxels by Using Perfusion Magnetic Resonance Imaging for Cervical Cancer during Radiotherapy [J]. J Cancer Sci Ther, 2018, 4 (9): 254-259.

[28] Lu D. Qian J. et al. Expression of PTEN and survivin in cervical cancer: promising biological markers for early diagnosis and prognostic evaluation [J]. Br J Biomed, 2020, 69 (4): 143-6.

[29] Wu SF. Zhang JW. et al. Altered expression of survivin, Fas and FasL contributed to cervical cancer development and metastasis [J]. Eur Rev Med Pharmacol, 2018, 16 (15): 2044-50. 